\title{
Effects of constraint-induced movement therapy in children with hemiplegia: a single case experimental study
}

\section{Efeitos da terapia de restrição por movimento induzido em crianças com hemiplegia: desenho experimental de caso único}

\author{
Marina B. Brandão', Marisa C. Mancini², Daniela V. Vaz ${ }^{3}$, Ângela M. Bueno ${ }^{4}$, Sheyla R. C. Furtado ${ }^{3}, Z^{2}$ élia A. C. Coelho²
}

\begin{abstract}
Objective: To investigate the profile of changes in the use of the upper extremity in three children with hemiplegia submitted to an adapted protocol of constraint-induced movement therapy (CIMT). Methods: A single-subject design (ABA) was replicated in three children aged 8 to 11 years old. Baseline phases (A1) and (A2) and the intervention phase (B) lasted 2 weeks each. During the intervention period, children wore a splint on the non-affected extremity for 10 hours a day and were submitted to 3 hours of therapy a day during 10 days. Training consisted of activities with the affected upper extremity, with gradually increasing complexity and verbal feedback. Hand function was classified according to the Manual Ability Classification System (MACS). Children were assessed four times every week with the Toddler Arm Use Test (TAUT) and three adapted tasks from the Jebsen-Taylor Hand Function test (JTHF), and once a week with the Pediatric Motor Activity Log (PMAL) and self-care scales of the Pediatric Evaluation of Disability Inventory (PEDI). Celeration Line, Two-Standard Deviation Band and visual analysis methods were used for data analyses. Results: Significant improvements in the amount and quality of upper extremity use (PMAL), TAUT quality of use for children 2 and 3 , and participation for child 1, as well as decreased time to complete JTHF tasks for children 2 and 3 were observed. No changes were observed in the PEDI self-care scales. Conclusion: CIMT effects were associated with improvements in manual dexterity, amount and quality of use of the affected upper extremity in children with hemiplegia.
\end{abstract}

Key words: constraint therapy; hemiplegia; cerebral palsy; manual function.

\section{Resumo}

Objetivo: Investigar mudanças longitudinais no uso da extremidade superior em três crianças com hemiplegia submetidas a um protocolo adaptado de terapia de movimento induzido por restrição (CIMT). Métodos: Um desenho experimental de caso único (ABA) foi replicado em três crianças entre 8 e 11 anos de idade. Fases de baseline (A1) e (A2) e fase de intervenção (B) duraram duas semanas cada. Durante a fase de intervenção, as crianças usaram um splint na extremidade não afetada por dez horas por dia e foram submetidas a três horas de terapia diária por dez dias. O treinamento consistiu em atividades para a extremidade superior acometida, com aumento gradual da complexidade da tarefa e reforço verbal. A função manual foi classificada de acordo com o Manual Ability Classification System (MACS). As crianças foram avaliadas quatro vezes por semana com o Toddler Arm Use Test (TAUT) e três provas adaptadas do Teste Jebsen-Taylor de Função Manual (JTHF) e uma vez por semana com o Pediatric Motor Activity Log (PMAL) e escalas de autocuidado do Inventário de Avaliação Pediátrica de Incapacidade (PEDI). Os métodos Celeration Line, Banda de Dois Desvios-Padrão e análise visual foram usados para análise de dados. Resultados: Resultados significativos demonstraram melhora na qualidade e frequência de uso da extremidade superior (PMAL), qualidade de uso do TAUT nas crianças 2 e 3 , participação na criança 1, bem como diminuição do tempo gasto para completar as tarefas do JTHF para as crianças 2 e 3. Nenhuma mudança foi observada nas escalas de autocuidado do teste PEDI. Conclusão: Efeitos da CIMT associaram-se à melhora na destreza manual, frequência e qualidade de uso da extremidade acometida em crianças com hemiplegia.

Palavras-chave: terapia de restrição; hemiplegia; paralisia cerebral; função manual.

Received: 14/10/2008 - Revised: 13/03/2009 - Accepted: 05/05/2009

\footnotetext{
Universidade FUMEC, Associação Mineira de Reabilitação (AMR), Belo Horizonte (MG), Brazil

2 Occupational Therapy Department, Universidade Federal de Minas Gerais (UFMG), Belo Horizonte (MG), Brazil

${ }^{3}$ Physical Therapy Department, UFMG

${ }^{4}$ Occupational Therapist

Correspondence to: Marisa Cotta Mancini, Departamento de Terapia Ocupacional, Escola Educação Física, Fisioterapia e Terapia Ocupacional, Universidade Federal de Minas Gerais,

Campus Pampulha, Av. Antônio Carlos, 6.627, CEP 31270-910, Belo Horizonte (MG); Brazil, e-mail: mcmancini@pib.com.br
} 


\section{Introduction $: \therefore$.}

Children with hemiplegia have unilateral involvement of upper and lower extremities opposite to the side of cerebral injury, often characterized as muscle weakness, spasticity and hypertonia ${ }^{1}$. These factors may decrease movement efficiency ${ }^{2}$, especially in the use of the upper extremity, which can also limit performance in functional activities at home and school ${ }^{1,3,4}$.

Constraint-induced movement therapy (CIMT) has been used to promote functional gains in individuals with neurological dysfunctions ${ }^{5-11}$. CIMT consists of constraining movement of the non-affected upper extremity and providing intensive training to the involved upper extremity ${ }^{3,5,12}$. Intensive training is based on shaping principles ${ }^{3,5,7}$, which include the selection of activities suited to the client's individual abilities, with progressive increase in difficulty and complexity. Procedures also involve providing assistance and support when the individual is unable to perform the task independently, as well as verbal rewards for observed improvements ${ }^{9}$. One of the main objectives of the intervention is to overcome the learned nonuse, defined as the diminished use of the affected extremity due to the perception of failure during the performance of manual tasks ${ }^{13,14}$.

The original CIMT protocol consists of 2 or 3 weeks of daily intensive training of the affected extremity for 6 hours in association with restriction of the non-affected extremity for 10 hours a day ${ }^{13}$. According to Gordon, Charles and Wolf ${ }^{3}$, the original CIMT protocol consisting of six hours of daily training and use of restraint for $90 \%$ of waking hours could be tiring for children. Thus, modifications in specific aspects of the original CIMT protocol have been proposed by some authors ${ }^{2,6,14-16}$. These adaptations include decreased training time and/or decreased use of the restriction ${ }^{15-18}$ which are often compensated for with increased protocol lengths ${ }^{6}$. Charles et al. ${ }^{16}$ investigated a protocol in which the use of the restriction occurred only during the intensive training of 6 hours daily, for 12 days. They documented significant improvements in children's manual dexterity and parents reported improvements in the amount and quality of use of the affected extremity ${ }^{16}$. Eliasson et al. ${ }^{6}$ studied the effects of a two-month intervention protocol with two hours of training and restriction use every day in young

Table 1. Descriptive analysis of the three children in terms of age, sex and MACS level.

\begin{tabular}{lccc}
\hline Participant & Age & Sex & MACS level $^{*}$ \\
\hline Child 1 & 8 years old & female & II \\
\hline Child 2 & 11 years old & female & III \\
\hline Child 3 & 8 years old & male & I
\end{tabular}

*MACS: Manual Ability Classification System which includes five levels; level I indicates no difficulty to use the hands to perform daily activities, level II indicates that use of the hands in daily activities shows lower quality and/or takes longer, level III informs that child shows difficulty in using hands and needs help to set-up for activity performance and/or requires modification of activity. children. The authors reported positive results with significant improvements in children's bimanual abilities ${ }^{6}$.

The replication of available evidence in a different cultural environment involves testing the effects of this intervention considering the clinical particularities of the Brazilian children, as well as our rehabilitation services. The investigation of the feasibility of adapted CIMT models to meet Brazilian children's needs and promote improvements in manual dexterity and daily functioning may help broaden clinical actions of rehabilitation professionals. The objective of this study was to investigate the profile of changes in the functional use of the upper extremity in three children with hemiplegia submitted to an adapted CIMT protocol. The study's hypotheses were: $\mathrm{H} 1$ : there will be improvements in the quality and in the amount of use of the affected extremity in children with hemiplegia, after administration of an adapted CIMT protocol; H2: there will be improvements in the manual dexterity of children with hemiplegia submitted to an adapted CIMT protocol; H3: there will be improvements in daily functioning of children with hemiplegia after the administration of an adapted CIMT protocol.

\section{Method $: \because$.}

\section{Study design}

An ABA experimental single-subject design replicated in three children was conducted. Baseline phases (A1) and (A2) consisted of repeated assessments without treatment. Phase B consisted of CIMT intervention and periodic assessments. Each phase lasted two weeks, totaling six weeks of study.

\section{Participants}

Participants of this study had a medical diagnosis of spastic hemiplegic cerebral palsy or stroke, and had cognitive abilities to follow verbal commands. They had no associated pathologies or movement disorders, did not make use of hand splints or received botulinum toxin in the upper extremity within six months prior to the beginning of the study. During the study, children were not receiving other interventions to improve upper extremity function. The three children were contacted by convenience by one of the investigators. They attended rehabilitation services in Belo Horizonte/Brazil, and agreed, with permission from their parents, to participate in this study. They all conformed to the inclusion criteria described above. Table 1 presents descriptive information of each child.

Child 1 was a girl with right hemiplegia due to cerebral palsy. She demonstrated diminished use of the right upper extremity in daily activities, weakness of the intrinsic hand muscles and 
difficulty to perform tasks that required manual dexterity. This child was discharged from occupational therapy services one month before the beginning of this study.

Child 2 was a girl with right hemiplegia due to stroke at age 9. Her thumb was adducted and her wrist was flexed at rest, and wrist extensors and intrinsic hand muscles demonstrated weakness. Her occupational and physical therapy sessions were interrupted two weeks prior to the beginning of the study.

Child 3 was a boy with right hemiplegia due to cerebral palsy. He was able to use the affected upper extremity in bimanual activities, but had difficulties with finger movement dissociation in tasks demanding fine motor coordination. He had not received any therapeutic intervention since he was six years old.

\section{Instruments and procedures}

The study was approved by the Ethics Review Committee from the Universidade Federal de Minas Gerais (ETIC 147/05) and parents signed a consent form authorizing the child's participation. Before data collection, researchers were trained in the application of all instruments. Test-retest reliability assessed with Intraclass Correlation Coefficients (ICC) varied between 0.73 and 0.99. Specific reliability values for each assessment are indicated below. The same evaluator, who did not take part in the intervention program (an occupational therapist), performed all the assessments throughout the study.

Upper extremity functional performance was assessed four times a week during the six weeks of the study. Hand function was classified by observation of each child performing typical daily tasks, according to the Manual Ability Classification System $(\mathrm{MACS})^{19}$, which provides five categories, varying from complete dependence to perform manual activities (level V), to the use of both hands independently and without difficulty (level I). Hand dexterity was assessed with three manual tasks based on the Jebsen Taylor Hand Function Test (JTHF) ${ }^{20-22}: 1$ ) picking up small objects, 2) stacking four wooden discs, 3) picking up five round containers. The time to complete each task, as well as the total time to complete the three tasks was used for analyses. In the present study, JTHF reliability coefficients varied from 0.86 to 0.96 .

Functional use of the affected upper extremity was evaluated with the Toddler Arm Use Test (TAUT) ${ }^{5}$ four times a week throughout the study, totaling 24 administrations of this test. The TAUT is a standardized observational test that includes 21 functional activities rated according to the child's willingness to use the affected extremity, the amount of participation and the quality of movement of the affected extremity ${ }^{5}$. Children were videotaped during the assessments and after the end of data collection the images were scored in random order by consensus between two examiners blind to the study phases. TAUT ICC varied from 0.73 to 0.99 .
Functioning in daily living activities was assessed for descriptive purposes with the Pediatric Motor Activity Log $(\mathrm{PMAL})^{5}$ and the Pediatric Evaluation of Disability Inventory $(\mathrm{PEDI})^{23}$. Frequency of administration of each test was once a week (i.e., twice in each phase), totaling six assessments throughout the study. The PMAL is a semi-structured interview with the child's caregiver. Caregiver's perceptions about the amount and quality of use of the child's affected upper extremity in 22 typical daily living activities were scored on a 0 to 5 scale $^{5}$. A Portuguese-translated version of the PMAL was used for data collection. PMAL ICC varied from 0.75 to 0.90 . The PEDI consists of an interview with the child's caregivers and informs about functioning in daily living tasks ${ }^{23}$. For the present study, only self-care functional skills and caregiver assistance scales were used. This test is available in a Portuguese version adapted to Brazilian cultural specificities ${ }^{24}$ and is valid and reliable ${ }^{25}$. PEDI test-retest ICC was 0.99 .

During phase B, children were submitted to an adapted CIMT protocol with three hours of functional training a day, five days a week, for two weeks. This period was arranged to happen during school holidays to allow for better organization of children's schedules and avoid interfering with their participation at school. Many authors are investigating adapted intervention protocols that better conform to children's particularities ${ }^{2}$. In an attempt to develop a more child-friendly version of this intervention, adapted for the Brazilian clinical reality, our procedure consisted of decreasing the time spent in therapy to three hours a day in association with constant caregiver input indicating activities that would be interesting for children to perform at home. Moreover, in spite of maintaining the use of the restriction throughout the day, restriction devices were included progressively. In the first week of intervention, children used only a ventral thermoplastic splint that immobilized wrist and finger movements. In the beginning of the second week, the elbow and shoulder were restricted with the neck sling. Thus, children and caregivers had time to adapt to the use of restriction progressively.

Functional training was provided by two occupational therapists and a physical therapist. Each child was assisted by one therapist and performed individual and group activities. The intervention schedule included functional activities (dressing, eating, brushing teeth, preparing meals), fine motor activities, arts, board and card games and gross motor function games. Intervention aimed at stimulating the use of the affected upper extremity during performance of self-care and play activities. Specific activities involving reaching, prehension, in-hand manipulation, and object release were selected based on the child's interests and goals established by the therapist ${ }^{5}$. In accordance with the principles of shaping, complexity and difficulty of tasks were progressively increased during training ${ }^{9,16}$. Tasks were 
broken down into simpler components as needed, and the level of complexity and difficulty of the tasks was gradually increased with greater demands for precision, velocity, and versatility. As children demonstrated improvements, they received positive verbal feedback and rewards ${ }^{5}$. During intervention, shaping procedures (i.e., grading of task's complexity and verbal feedback/ rewards) and the use of group activities contributed to create a motivating environment in which children were enthusiastic to use the affected extremity. The restraint was used during the sessions without interruption only for the intervention phase (B). Parents were instructed to keep a daily log of the time of restriction use at home, the activities performed during the day, as well as difficulties and improvements observed at home. This log was checked daily by the therapists in order to monitor and ensure children's adequate use of the restriction at home.

\section{Data analyses}

The Two-Standard Deviation Band (TSDB) or the Celeration Line (CL) methods were used to analyze results from the TAUT and dexterity tests. The CL compares the rate of change between consecutive phases through the determination of a trend line ${ }^{26}$. Differences in the tendency of change of performance with the introduction of a new study phase is tested according to the proportion of data points above and below the

Table 2. Results of the Two-Standard Deviation Band Method or Celeration Line Method to analyze JTHF test (tasks 1, 2, 3 and total) and TAUT test (quality, participation and willingness scales) of the three children, in the A1-B (baseline $x$ intervention) and B-A2 (intervention $x$ post-intervention) periods.

\begin{tabular}{|c|c|c|c|c|c|c|}
\hline \multirow{2}{*}{ Phases } & \multicolumn{3}{|c|}{$A 1-B$} & \multicolumn{3}{|c|}{ B-A2 } \\
\hline & Child 1 & Child 2 & Child 3 & Child 1 & Child 2 & Child 3 \\
\hline $\begin{array}{l}\text { JTHF } \\
\text { Task } 1\end{array}$ & ns & ns & ns & ns & * & * \\
\hline $\begin{array}{l}\text { JTHF } \\
\text { Task } 2\end{array}$ & ns & ns & ns & ns & $\mathrm{nS}$ & ns \\
\hline $\begin{array}{l}\text { JTHF } \\
\text { Task } 3\end{array}$ & ns & ns & ns & ns & ns & ns \\
\hline $\begin{array}{l}\text { JTHF } \\
\text { Total time }\end{array}$ & ns & * & ns & ns & * & ns \\
\hline $\begin{array}{l}\text { TAUT } \\
\text { Quality }\end{array}$ & * & * & ** & ns & ns & ** \\
\hline $\begin{array}{l}\text { TAUT } \\
\text { Participation }\end{array}$ & * & ns & ns & * & ns & ns \\
\hline $\begin{array}{l}\text { TAUT } \\
\text { Willingness }\end{array}$ & ns & ns & ns & ns & ns & ns \\
\hline
\end{tabular}

ns $=$ non-significant result; ${ }^{*}=$ significant result in the expected direction (JTHF: decrease in time to perform the test, TAUT: increase in the score); ${ }^{* *}=$ significant result in the opposite expected direction (TAUT: decrease in the score). Note: The Two-Standard Deviation Band was used to analyze results from the JTHF tests and TAUT scales, except for the total score in JTHF from child 2, and for the scores of quality scale for children 1 and 3 , which the Celeration Line Method was used. trend line of the previous phase with the Binomial test. Level of significance was set to $\alpha=0.05^{26}$. The TSDB is appropriate when there are no significant trends, as tested with the C-Statistics, or auto-correlation in baseline data ${ }^{27,28}$. According to the TSDB, the magnitude of change is considered significant if at least two consecutive data points fall above or below two standard deviations from the mean of the previous phase ${ }^{26}$.

Results from PMAL and PEDI tests were visually analyzed according to the level, direction of change and slope of data points across phases ${ }^{26}$. Because of the small number of data points, statistical analysis was not performed and these data were used descriptively.

\section{Results $: \because$.}

The TSDB was used to analyze dexterity data, except for total time for child 2, which was analyzed with CL used because of auto-correlation observed in the baseline (Table 2).

According to the daily logs, parents reported that children used the sling and the splint in their home environment during the two weeks of intervention, despite initial reluctance to use the restriction device in the first week of the intervention phase. Parents reported that the use of restriction by the participants lasted, on average, 10 hours a day.

\section{Child 1}

Child 1 demonstrated significant improvements in TAUT quality of use and participation scales between Al and B assessments. No changes were observed in the JTHF test or in the TAUT willingness of use scale. Visual analysis revealed pronounced trends towards gains in PMAL amount and quality of use scales. Between phases B and A2, Child 1 improved in the TAUT participation scale. No changes were observed in the JTHF test and in the TAUT quality of use and willingness of use scale. Visual analysis demonstrated pronounced trends towards improvements in the PMAL quality scale and maintenance of improvements in PMAL amount of use scale. No important differences were observed in the PEDI scales (Figure 1).

After intervention, Child l's mother reported functional improvements. The girl demonstrated important improvements in domestic tasks involving bimanual abilities (i.e. washing up, folding clothes).

\section{Child 2}

Child 2 demonstrated a significant decrease in the total time to perform the JTHF tasks and significant improvements in the 


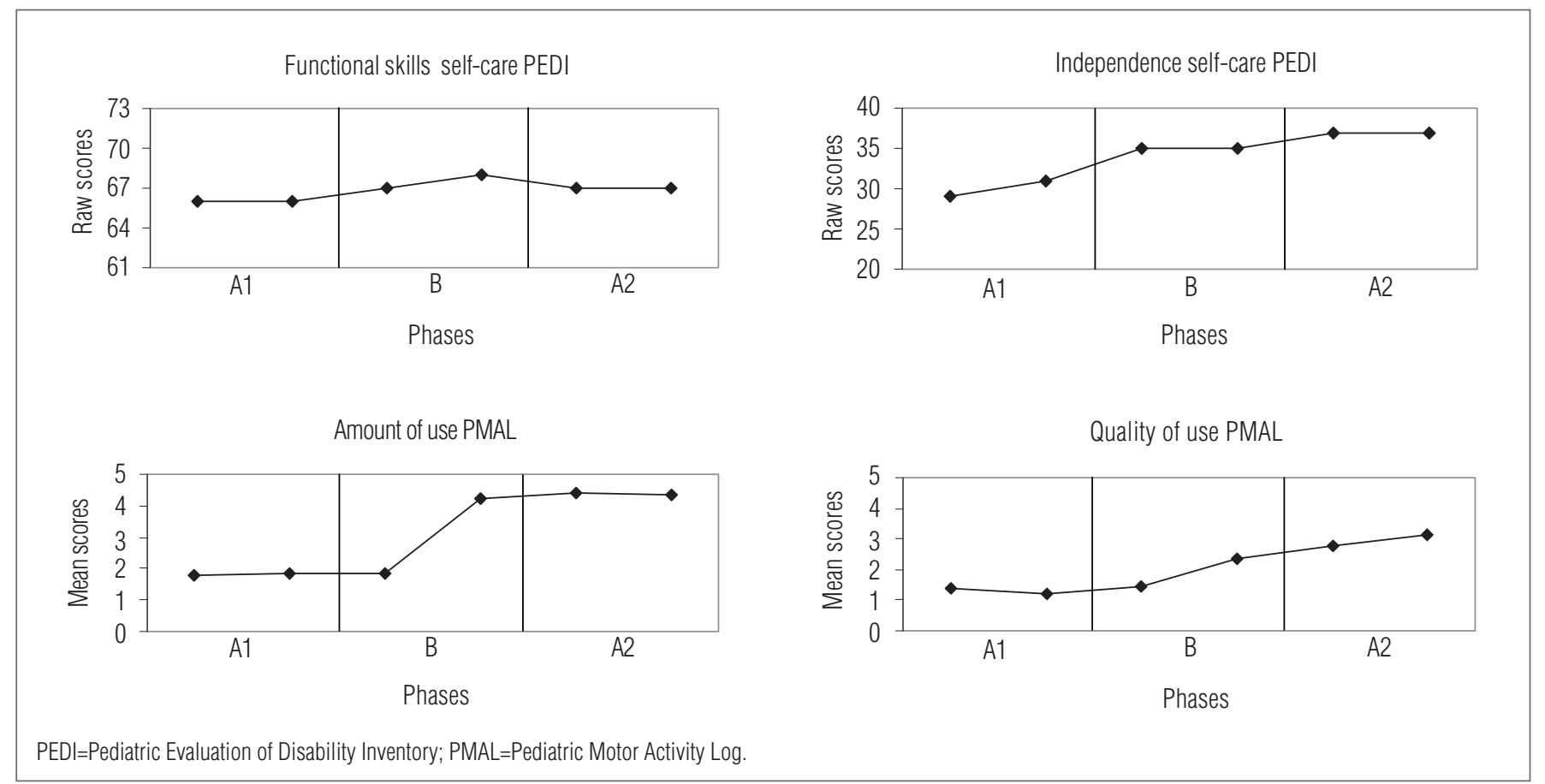

Figure 1. Raw scores of functional skills and independence in self-care from the PEDI and mean scores of quality of use and amount of use scales from PMAL in the A1 (baseline), B (intervention) and A2 (post-intervention) phases of Child 1.

TAUT quality of use scale, between phases A1-B. No statistically significant change was observed in the TAUT participation or willingness scale. The visual analysis revealed pronounced trends towards improvements in the PMAL amount of use and quality of use scales. When phase A2 was compared to phase B, Child 2 demonstrated a significant decrease in the time to perform JTHF Task 1 and Total Time. No changes were observed in the TAUT scales. The visual analysis demonstrated a slight trend towards decrease in the PMAL frequency of use scale, but the scores were still higher than phase A1. Child 2 maintained the previous gains in the PMAL quality of use scale. No important differences were observed in the PEDI scales (Figure 2).

After the intervention period, parents reported that Child 2 demonstrated improved prehension of objects during recreational activities, more awareness of the possibility of using the affected hand during play and school activities and less need of assistance.

\section{Child 3}

No significant improvements were found in the JTHF and TAUT quality and willingness of use scales when phase B was compared to A1. Visual analysis revealed a pronounced trend towards increase in the PMAL amount of use scale. No changes were observed in the PMAL quality of use scale. When phase A2 was compared to phase B, Child 3 demonstrated a significant decrease in the time to perform JTHF Task 1. No changes were observed in the TAUT participation and willingness of use scales. Visual analysis showed a slight trend towards decrease in the PMAL amount of use scale and maintenance of gains in the quality of use scale. No changes were observed in the PEDI scales because the child presented maximum scores from the beginning of the study. Child 3 demonstrated a significant decrease in the TAUT quality of use scale in both A1-B and B-A2 periods. However, the visual analysis does not conform to this profile, as it presents a stable trend of data (Figure 3).

According to Child 3's mother, he improved the ability to grasp small objects and was willing to use the affected extremity spontaneously during self-care and play activities.

\section{Discussion $: \because:$}

Results from the present study show improvements in quality and amount of use of the affected upper extremity following CIMT in two of the three children with hemiplegia. Despite a general trend towards improvement in dexterity, statistical methods detected significant gains in only $16 \%$ of the comparisons. Based on the small effects observed in two children, it is not possible to conclude that the protocol of three hours of daily training may effectively enhance manual dexterity in children with hemiplegia, not confirming H2. The lack of improvements in the adapted JTHF test contrasts with the findings of Charles et al. ${ }^{16}$. In their study, the authors observed significant improvements in the JTHF test for children submitted to the CIMT protocol. Differences in 
Functional skills self-care PEDI

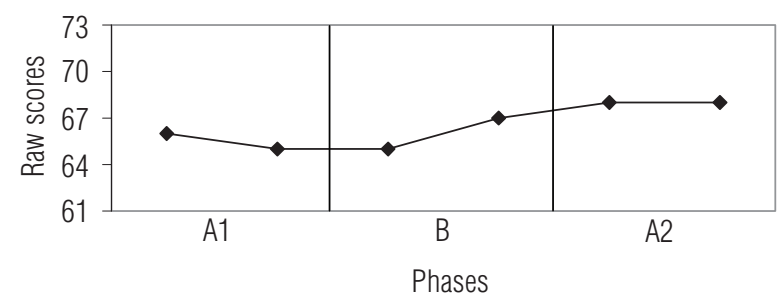

Amount of use PMAL

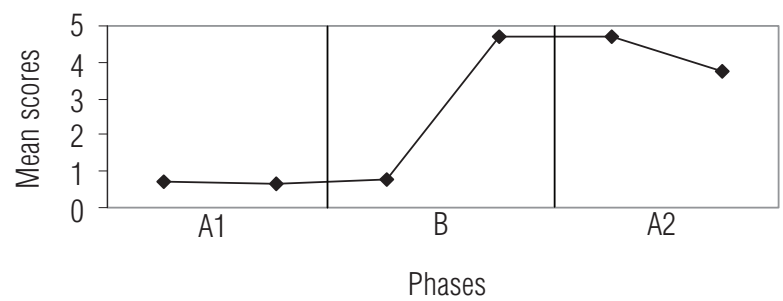

Independence self-care PEDI

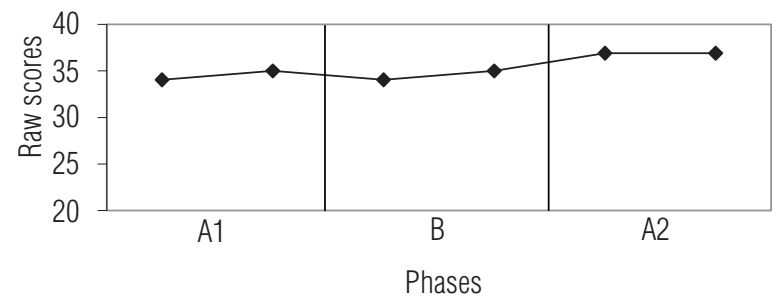

Quality of use PMAL

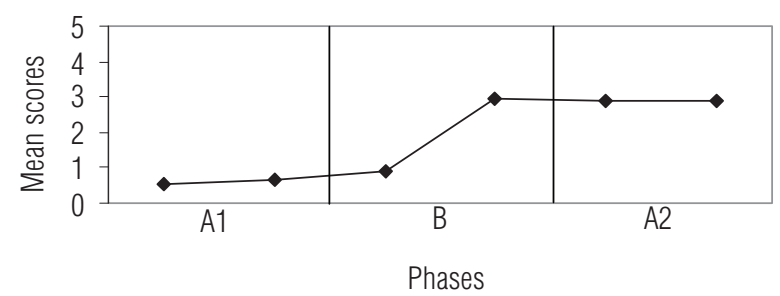

PEDI=Pediatric Evaluation of Disability Inventory; PMAL=Pediatric Motor Activity Log.

Figure 2. Raw scores of functional skills and independence in self-care from the PEDI and mean scores of quality of use and amount of use scales from PMAL in the A1 (baseline), B (intervention) and A2 (post-intervention) phases of Child 2.

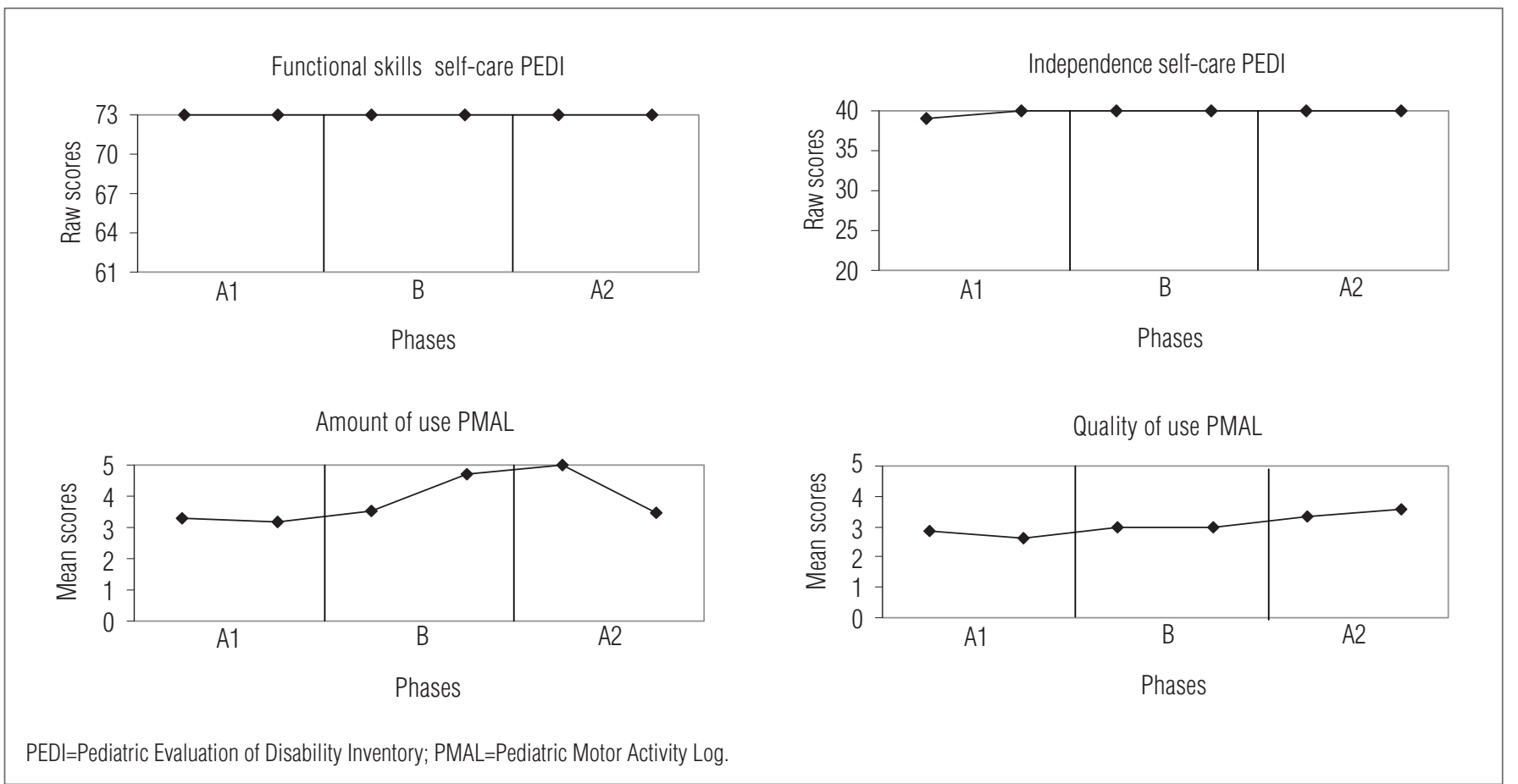

Figure 3. Raw scores of functional skills and independence in self-care from the PEDI and mean scores of quality of use and amount of use scales from PMAL in the A1 (baseline), B (intervention) and A2 (post-intervention) phases of Child 3.

protocol intensity between studies could explain this discrepancy in results. In their study, Charles et al. ${ }^{16}$ conducted training for six hours a day, while in the present study the training program lasted 3 hours. Thus, it is argued that the decreased intensity protocol used in the present study was not sufficient to promote improvements in children's manual dexterity.

Significant improvements in quality of use of the affected extremity, as hypothesized in $\mathrm{H} 1$, were observed in two children. The gains in quality of use of the affected extremity and the lack 
of significant improvements in manual dexterity in most tasks could be interpreted as contradictory results. However, the lack of significant decrease in time spent to perform tasks could be a consequence of the improvement in quality of use of the affected extremity. As children experience improved movement quality, they develop better strategies to manipulate objects. These new abilities, however, are still emerging and performance is not yet mastered, which could have led to increased time to perform specific tasks. Long-term follow-up is needed to document the amount of time required for stable performance following this type of training, and whether or not such stabilization is followed by improvements in manual dexterity.

No changes were observed for the TAUT willingness and participation scales, except for child 1, who demonstrated improved participation scores during intervention and in the post-intervention phases. The lack of changes in the other children could be attributed to the high scores presented at baseline (A1); they received the maximum score (score 2) and maintained this level throughout the study. These high scores in the TAUT willingness and participation scales and the low scores in the PMAL amount of use of the affected extremity, at the baseline period, illustrate the learned nonuse phenomenon. In the current study, despite the fact that children did not present any resistance to use the affected extremity in clinical environment, parents reported low frequency of use of the affected upper extremity in the home environment.

According to parents' perceptions documented with the PMAL, all children demonstrated improvements in the amount and quality of use of the affected extremity in functional activities during the intervention period, with maintenance of the quality scores and a small decrease in frequency of use in the follow-up period. This small decrease could be explained by the return of use of the non-affected extremity during performance of functional activities. The overall positive results presented in PMAL scores highlight the principles of CIMT. According to Kunkel et al..$^{29}$, one of the greatest benefits of CIMT would be the decrease in the difference between current performance and real potential for the execution of daily activities, as the intervention is directed to overcome the learned nonuse.

PMAL results, however, should be interpreted with caution. Statistical analysis could not be undertaken in the current study, due to the small number of assessments. We considered that repetition of the interview four times a week could lead parents to overestimate their child's performance during and after intervention, and chose to perform only one interview each week in order to minimize the possible influence of repeated measurements as a threat to the study's internal validity. In the present study, the positive results documented by the TAUT quality scale corroborate the improvements in quality and the amount of use of the affected extremity reported in PMAL interviews.

The lack of functional improvements in the PEDI (H3) seems to be due to the functional characteristics of the children included in the study, as they obtained high scores from the beginning of baseline. In spite of the lack of significant results in PEDI, parents reported improvements in the performance of daily living activities, documented in the daily logs. Future studies should investigate the effects of CIMT on functional abilities and independence, among younger children or children with different levels of manual function.

According to the results of this study, the use of CIMT was effective to promote improvements in the amount and quality of use of the affected upper extremity during functional activities in two of the three children with hemiplegia. Future studies analyzing children's and parents' perceptions regarding the training program and the use of restraint would help to further elucidate the benefits and applicability of traditional and adapted CIMT protocols in children. This has been the first Brazilian study to investigate the use of an adapted CIMT model for children with $\mathrm{CP}$, however, it is important and necessary to continue testing this intervention in our clinical population in order to validate our results. Such information is of great relevance for clinical practice, considering the particularities of health services which call for an adaptation of the technique in order to favor parent and therapist adhesion to the intervention.

\section{Acknowledgments : :}

This study was supported by grants from two Brazilian government agencies: Conselho Nacional de Desenvolvimento Científico e Tecnológico (CNPq), and Fundação de Apoio à Pesquisa do Estado de Minas Gerais (FAPEMIG). We also thank the collaboration of Luciana Braga and Rafaela Alvarenga, who helped during the administration of the intervention. 


\section{References}

1. World Health Organization. International classification of functioning, disability and health (ICF). Geneva: World Health Organization; 2001.

2. Charles J, Gordon AM. A critical review of constraint-induced movement therapy and forced use in children with hemiplegia. Neural Plast. 2005;12(23):245-61.

3. Gordon AM, Charles J, Wolf SL. Methods of constraint-induced movement therapy for children with hemiplegic cerebral palsy: development of a childfriendly intervention for improving upper-extremity function. Arch Phys Med Rehabil. 2005;86(4):837-44.

4. Eliasson AC, Bonnier B, Krumlinde-Sundholm L. Clinical experience of constraintinduced movementtherapy inadolescents with hemiplegic cerebral palsy - a day camp model. Dev Med Child Neurol. 2003;45(5):357-9.

5. Taub E, Ramey SL, DeLuca S, Echols K. Efficacy of constraint-induced movement therapy for children with cerebral palsy with asymmetric motor impairment. Pediatrics. 2004;113(2):305-12.

6. Eliasson AC, Krumlinde-Sundholm L, Shaw K, Wang C. Effects of constraintinduced movement therapy in young children with hemiplegic cerebral palsy: an adapted model. Dev Med Child Neurol. 2005;47(4):266-75.

7. Liepert J, Bauder H, Wolfgang HR, Miltner WH, Taub E, Weiller C. Treatment-induced cortical reorganization after stroke in humans. Stroke. 2000;31(6):1210-6.

8. Sterr A, Elbert T, Berthold I, Kolbel S, Rockstroh B, Taub E. Longer versus shorter daily constraint-induced movement therapy of chronic hemiparesis: an exploratory study. Arch Phys Med Rehabil. 2002;83(10):1374-7.

9. Taub E, Uswatte G, Pidikiti R. Constraint-induced movement therapy: a new family of techniques with broad application to physical rehabilitation - a clinical review. J Rehabil Res Dev. 1999;36(3):237-51.

10. Pierce SR, Daly K, Gallagher KG, Gershkoff AM, Schaumburg SW. Constraint-induced therapy for a child with hemiplegic cerebral palsy: a case report. Arch Phys Med Rehabil. 2002;83(10):1462-3.

11. DeLuca SC, Echols K, Ramey SL, Taub E. Pediatric constraint-induced movement therapy for a young child with cerebral palsy: two episodes of care. Phys Ther. 2003;83(11):1003-13.

12. Miltner WH, Bauder H, Sommer M, Dettmers C, Taub E. Effects of constraintinduced movement therapy on patients with chronic motor deficits after stroke: a replication. Stroke. 1999;30(3):586-92.

13. DeLuca SC, Echols K, Law CR, Ramey SL. Intensive pediatric constraintinduced therapy for children with cerebral palsy: randomized controlled, crossover trial. J Child Neurol. 2006;21(11):931-8.

14. Sterr A, Freivogel S, Schmalohr D. Neurobehavioral aspects of recovery: assessment of the learned nonuse phenomenon in hemiparetic adolescents. Arc Phys Med Rehabil. 2002;83(12):1726-31.
15. Charles J, Lavinder G, Gordon AM. Effects of constraint-induced therapy on hand function in children with hemiplegic cerebral palsy. Pediatr Phys Ther 2001;13(2):68-76.

16. Charles JR, Wolf SL, Schneider JA, Gordon AM. Efficacy of a child-friendly form of constraint-induced movement therapy in hemiplegic cerebral palsy: a randomized control trial. Dev Med Child Neurol. 2006;48(8):635-42.

17. Naylor CE, Bower E. Modified constraint-induced movement therapy for young children with hemiplegic cerebral palsy: a pilot study. Dev Med Child Neurol. 2005;47(6):365-9.

18. Gordon A, Connelly A, Neville B, Vargha-Khadem F, Jessop N, Murphy T, et al. Modified constraint-induced movement therapy after childhood stroke. Dev Med Child Neurol. 2007;49(1):23-7.

19. Eliasson AC, Krumlinde Sundholm L, Rösblad B, Beckung E, Arner M, Öhrvall AM, et al. The manual ability classification system (MACS) for children with cerebral palsy: scale development and evidence of validity and reliability. Dev Med Child Neurol. 2006;48(7):549-54.

20. Jebsen RH, Taylor N, Trieschmann RB, Trotter MJ, Howard LA. An objective and standardized test of hand function. Arch Phys Med Rehabil. 1969;50(6):311-9.

21. Taylor N, Sand PL, Jebsen RH. Evaluation of hand function in children. Arch Phys Med Rehabil. 1973;54(3):129-35.

22. Vaz DV, Mancini MC, Fonseca ST, Vieira DS, de Melo Pertence AE. Muscle stiffness and strength and their relation to hand function in children with hemiplegic cerebral palsy. Dev Med Child Neurol. 2006;48(9):728-33.

23. Haley SM, Coster WJ, Ludlow LH, Haltiwanger JT, Andrellow PJ. Pediatric evaluation of disability inventory: development, standardization and administration manual. Boston: New England Medical Center; 1992.

24. Mancini MC. Inventário de avaliação pediátrica de incapacidade (PEDI) manual da versão brasileira adaptada. Belo Horizonte: UFMG; 2004.

25. Feldman AB, Haley SM, Coryell J. Concurrent and construct validity of the pediatric evaluation of disability inventory. Phys Ther. 1990;70(10): 602-10.

26. Portney LG, Watkins MP. Foundations of clinical research: applications to practice. $2^{a}$ ed. New Jersey: Prentice Hall Health; 2000.

27. Ottenbacher KJ. Evaluating clinical change: strategies for occupational and physical therapists. Baltimore: Williams \& Wilkins; 1986.

28. Nourbakhsh MR, Ottenbacher KJ. The statistical analysis of single-subject data: a comparative examination. Phys Ther. 1994;74(8):768-76.

29. Kunkel A, Kopp B, Muller G, Villringer K, Villringer A, Taub E, et al. Constraint-induced movement therapy for motor recovery in chronic stroke patients. Arch Physical Med Rehabil. 1999;80(6):624-8. 